\title{
Producción de biocombustibles y empleo rural en Colombia 2009-2015
}

\author{
Jorge Barrientos Marín* \\ Carlos Andrés Vasco Correa**
}

Fecha de recepción: 5 de diciembre de 2019

Fecha de aceptación: 6 de mayo de 2020

Resumen: Este trabajo de investigación estima el efecto de la política de mezcla de biocombustibles sobre la generación de empleo rural en Colombia, a través de la aplicación de la metodología empírica basada en vectores autorregresivos (VAR), para estimar la elasticidad del nivel de empleo y simular su comportamiento cuando la producción y los precios de biocombustibles o combustibles fósiles sufren variaciones. Se construye una base de datos que contiene nivel de producción, precios y empleo con periodicidad mensual entre 2009 y 2015 en Colombia. Se estudia la estructura empírica subyacente y la manera como se puede estimar la elasticidad en modelos vectoriales, finalmente se analiza la política de promoción de los biocombustibles y sus efectos sobre la generación de empleo. Las correlaciones dinámicas estimadas muestran que no existe un efecto apreciable sobre el nivel de empleo cuando hay variaciones en los niveles de producción y precios de los biocombustibles.

Palabras clave: planificación económica; política económica; política energética; empleo; economía de la energía; biocombustibles; modelos VAR.

Clasificación JEL: L52, Q16, Q48, C52.

Cómo citar este artículo/ To reference this article / Comment citer cet article / Para citar este artigo:

Barrientos Marin, J. \& Vasco Correa, C. (2020). Producción de biocombustibles y empleo rural en Colombia 2009-2015. Apuntes del Cenes, 39 (70). Págs. 233 - 260. https://doi.org/10.19053/01203053.v39.n70.2020.10426

\footnotetext{
PhD. en Economía, profesor titular Universidad de Antioquia, Departamento de Economía. Medellín, Colombia Correo electrónico: jorge.barrientos@udea.edu.co iD https://orcid.org/0000-0001-5368-1928

** M.Sc. en Economía, profesor asistente Universidad de Antioquia, Departamento de Economía. Medellín, Colombia. Correo electrónico: carlos.vasco@udea.edu.co iD https://orcid.org/0000-0002-6050-0520
} 


\title{
Biofuel Production and Rural Employment in Colombia 2009-2015
}

\begin{abstract}
This paper estimates the effect of the biofuel mixing policy on the Colombian rural employment. To this end, we apply an empirical methodology based on autoregressive vectors -VAR-. This one allows us to estimate the short-run employment elasticity and to simulate its evolution when biofuel or its prices suffer a positive shock. We construct a monthly database that contains production, prices, and employment levels during 2009-2015 in Colombia. Underlying empirical structure and how elasticity can be estimated in vector models will be studied, and finally we analyze biofuel promotion policy and its effects on jobs. The estimated dynamic correlations show that there is no appreciable effect on the employment levels when there are variations in biofuels production and prices levels.
\end{abstract}

Keywords: economic planning, economic policy, energy policy, employment, energy economics, biofuels, VAR models. 


\section{INTRODUCCIÓN}

En Colombia desde el año 2005 se promueve la mezcla de combustibles de origen fósil como la gasolina corriente y el diésel, con combustibles de origen vegetal como el alcohol carburante y el biodiésel respectivamente. Con esta política de mezcla, el Estado pone en marcha una política pública con importantes objetivos estratégicos para el país: (i) incrementar la seguridad energética; (2) disminuir el consumo de energía no renovable y evitar su importación desde otros países; (3) mejorar la calidad del aire (el combustible fósil al mezclarse con biocombustibles optimiza su combustión); (4) promover la exportación de biocombustibles a medio plazo (en especial con aquellos que comparten la preocupación por el cuidado del aire y el cambio climático); (5) impulsar una agroindustria que dinamice las inversiones en el campo colombiano, modificando el uso del suelo principalmente (actualmente una gran parte dedicado a la ganadería extensiva) y (6), generar empleo y desarrollo económico en las zonas rurales del país.
El objetivo de este trabajo es establecer si el efecto de la política de mezcla de combustibles sobre los indicadores de empleo en las zonas rurales del país fue positivo entre 2009 y 2015 , puesto que si bien este es uno de los objetivos estratégicos del Gobierno nacional en el diseño de la política de promoción de biocombustibles, también se ha constituido en uno de los principales argumentos que tienen los productores del sector para apoyar su ampliación con mezclas más altas y continuar y profundizar la protección arancelaria frente a importaciones principalmente de Brasil y Estados Unidos.

Para lograr este objetivo se construyó en esta investigación una base de datos entre 2009 y 2015 que contiene como variables relevantes la producción de biocombustibles, los precios regulados y la producción de combustibles fósiles, etanol y biodiésel. Asimismo, se considera el precio del azúcar y el aceite de palma como medidas de costo de oportunidad, y los datos sobre el nivel de empleo asociado a la producción de azúcar y aceite de palma, como variables proxy de los niveles de contratación 
en los sectores de producción de etanol y biodiésel. Con esta información se estudian las correlaciones dinámicas entre las variables, estimando un sistema de ecuaciones a través de vectores autorregresivos (VAR).

Uno de los inconvenientes de usar series temporales de variables de producción y precios de combustibles en el mercado colombiano, es que su evolución temporal está determinada por la historia detrás de las variables, es decir, del modo en que diversas acciones exógenas de los agentes (como anuncios, leyes, regulaciones, eventos climáticos extremos) modifican su tendencia. En consecuencia, estas variables presentan cambios de nivel o quiebres estructurales que, en principio, implican que las variables podrían tener tendencias estocásticas impredecibles, lo que afecta los procedimientos de contraste de hipótesis de estacionariedad habituales como el test de Dickey-Fuller (Dickey \& Fuller, 1979) o el de Phillip-Perron (Phillips \& Perron, 1988). Es importante destacar que la aplicación de la metodología VAR requiere efectivamente la verificación de la estacionariedad de todas las variables involucradas en la estimación.

Como efectivamente ocurre en este estudio, muchas de las variables involucradas (en especial la producción de biocombustible y sus precios) presentan cambios de nivel que afectan el desempeño del contraste, lo que hace más probable que no se rechace la hipótesis nula de paseo aleatorio o no estacionariedad.
En virtud de esta situación, es necesario implementar una estrategia empírica alternativa para verificar estacionariedad. En esta investigación se utiliza la metodología de Cavaliere y Georgiev (2006), la cual controla bastante bien los cambios de nivel en las series involucradas, lo que conduce efectivamente a rechazar la hipótesis nula de raíz unitaria, entregando la evidencia necesaria para aplicar la metodología VAR. Algunos trabajos emplean esta metodología de manera exitosa, como por ejemplo Barrientos-Marin et al. (2018), donde se prueba que la serie mensual de precios de energía en bolsa en Colombia no tiene una raíz unitaria.

Una de las principales cualidades de los modelos VAR es la capacidad para simular el efecto de cambios en una variable sobre otras y además es un instrumento muy útil para obtener pronósticos a corto plazo si fuese el objetivo. El modelo VAR estimado en este trabajo incluye como variables producción, precio e índice de empleo asociado al etanol, además de una variable que refleja la producción de azúcar, con la cual el etanol comparte la misma materia prima. Evaluamos también un segundo modelo VAR que toma como variables el índice de empleo asociado al biodiésel, la producción y precio de este, incluyendo también la producción de aceite de palma, materia prima para la producción tanto de biodiésel como de productos del sector alimentos (aceites). Los resultados derivados de un exhaustivo análisis econométrico indican que 
tanto la producción de etanol como la de biodiésel interactúan con los respectivos índices de empleo, de tal manera que un shock a la producción de etanol, por ejemplo, trae como consecuencia un shock poco apreciable al índice de empleo asociado al etanol, con una relación positiva. La misma dinámica es cierta para la cadena del biodiésel.

Las contribuciones de este trabajo pueden resumirse en que, primero, hasta donde pudo establecerse, este es el primer artículo que indaga sobre la relación entre precio de biocombustibles y empleo en el sector agroindustrial de biocombustibles, utilizando una metodología de múltiples ecuaciones; segundo, está entre los pocos artículos en el ámbito nacional que estudia la estacionariedad de las variables con metodologías novedosas como la presentada por Cavaliere y Giorgiev (2006); tercero, los resultados obtenidos indican que no hay una causalidad entre el nivel de empleo en el sector y los cambios en los precios, una de las razones de muchos agentes para incentivar la producción nacional de biocombustibles y desincentivar las importaciones; y finalmente, los resultados encontrados pueden contribuir al debate sobre hasta dónde (y cuándo) es pertinente seguir aplicando la política de mezclas de fósiles con biocombustibles.

Este documento se divide en cinco secciones: en la primera, se presentan antecedentes del problema por tratar y una corta revisión literaria; en la segun- da, se explica con detalle la información estadística que se ha seleccionado para el desarrollo del trabajo; en la tercera, se presenta la metodología empleada; en la cuarta, se muestran los principales resultados obtenidos a partir de la estimación propuesta y en la quinta sección se concluyen y proveen algunas recomendaciones de política.

\section{ANTECEDENTES}

El empleo de biocombustibles ha sido un tema de especial atención en los últimos años por parte de los gobiernos alrededor del mundo, tanto por su pertinencia en el ámbito del desarrollo económico como por sus efectos de geopolítica y política energética. Diversos análisis e interrogantes han sido objeto de estudio en diferentes países como la Asociación de Países del Pacífico (APEC), la Unión Europea y América Latina; entre ellos, la pregunta que se plantea es cuál es el nivel de impacto que genera la producción de biocombustibles sobre los niveles de empleo rural. Una cuestión que no es en absoluto ajena a la realidad de Colombia, que desde el año 2001 se inserta en una política de promoción a la producción de biocombustibles a partir de la promulgación de la Ley 693, la cual se expidió bajo la premisa de que los biocombustibles son un factor que ayuda en tres temas: autosuficiencia energética, saneamiento ambiental, desarrollo y empleo rural (Congreso de Colombia, 2001). 
La producción de biocombustibles apoya y amplía la diversificación de la canasta energética colombiana, que solo cuenta con seis años de reservas de hidrocarburos tanto de gas natural como de petróleo (Ministerio de Minas y Energía, 2019), tiene una alta relevancia estratégica. Teniendo en cuenta la gran dependencia de los combustibles fósiles, es necesario promover el uso de los recursos naturales renovables y comenzar a producir combustibles más limpios, que además de reducir las emisiones de gases, protejan el medioambiente y el desarrollo rural.

Que la producción de biocombustibles haya aumentado de manera significativa en Colombia se debe en gran medida a la política de promoción que pusieron en marcha los gobiernos desde hace unos quince años. De esta manera, las poblaciones rurales que son dependientes del sector agropecuario se ven beneficiadas con las medidas tomadas al respecto por parte del Gobierno y están más interesadas en incrementar la producción de biocombustibles, ya que este tipo de actividades agroindustriales tiene de forma significativa como epicentro de desarrollo amplias zonas rurales.

Así pues, en Colombia, al igual que en otros países, el marco regulatorio es el principal mecanismo utilizado por el Gobierno para promover el desarrollo de los biocombustibles, a través del establecimiento de mezclas obligatorias e incentivos económicos a la inversión en el sector. A partir del diseño y ejecución de la política nacional de biocombustibles, estos productos han recibido un tratamiento preferente, principalmente por los ministerios de Minas y Energía y Hacienda, los cuales ofrecen acceso a regulación afín a la promoción del sector. También vale la pena reseñar que el etanol empezó a producirse en el país desde el 3 de octubre de 2005, y el biodiesel, el 1 de enero de 2008 (UPME, 2019).

Colombia posee grandes ventajas comparativas en cuanto a la disponibilidad de recursos naturales; así, en lo que tiene que ver con el sector de biocombustibles, según PROCOLOMBIA ${ }^{1}$ en el país se encuentran cerca de 7,4 millones de hectáreas disponibles para el desarrollo de agrocarburantes; asimismo, entre los principales productores de caña de azúcar, Colombia tiene la mayor tasa de rendimiento $(85,96$ ton/ ha), superando a Brasil (75,34 ton/ha), China (70,59 ton/ha), e India $(67,43$ ton/ ha). Colombia es el principal productor de palma de aceite en América Latina y el quinto en el mundo con más de 10 000 barriles diarios en seis refinerías y con una producción de etanol de 1,65 $\mathrm{m} / \mathrm{lt}$ por día en seis refinerías, convirtiéndose en el tercer productor de etanol en América Latina.

1 PROCOLOMBIA, antes PROEXPORT, es una agencia gubernamental de la rama ejecutiva del Gobierno de Colombia a cargo de promover las exportaciones colombianas, el turismo internacional y la inversión extranjera. 
Por lo anterior, la producción de biocombustibles puede generar grandes oportunidades para Colombia en materia de desarrollo económico, particularmente en reducción de emisión de gases efecto invernadero y en la generación de empleo; esto último se puede ver reflejado en cifras indicadas por la Federación Colombiana de Biocombustibles (FEDEBIOCOMBUSTIBLES). La agroindustria de los biocombustibles genera 28444 empleos directos y 150072 indirectos (FEDEBIOCOMBUSTIBLES, 2019). El $85 \%$ de dichos empleos, directos e indirectos, son atribuidos a la cadena agrícola, el resto a la cadena industrial. Existe entonces un enorme potencial en este sector para generar el empleo rural; por eso es pertinente investigar cuál es la incidencia a partir de contrastes estadísticos rigurosos.

El trabajo se realiza con fundamento en el análisis de datos desde los inicios de la implementación de la política en 2009 y durante un período de seis años hasta 2015, que incluye detalles sobre la efectividad de la política de incentivos a la promoción de la producción de combustibles más limpios y a la generación de empleo rural en Colombia. Además, se caracterizan de manera rigurosa las principales variables que inciden en la producción de los biocombustibles. Así, por ejemplo, los porcentajes de mezcla y los precios del etanol y el biodiésel son regulados por el Ministerio de Minas y Energía, por tanto, cualquier modificación sobre estos factores tendrá impac- tos sobre el comportamiento del sector, en lo referente a los niveles de producción, comercialización y consumo.

En un trabajo desarrollado por Sánchez (2015), se examina la evolución de los biocombustibles en Colombia y la incidencia en el precio de los alimentos. Se lleva a cabo un análisis estadístico y econométrico que muestra cómo la industria de biocombustibles incide de manera directa en el precio; sin embargo, no se incluyeron análisis respecto de la generación de empleo. Solo se contemplan, en el agregado, los resultados de datos estadísticos del Departamento Administrativo Nacional de Estadística (DANE), la encuesta continua de hogares (2002-2005) y la gran encuesta de hogares (2008-2012), las cuales muestran el desalentador panorama de desempleo y condiciones de pobreza en el sector rural. Lo anterior, a pesar de los grandes esfuerzos por mejorar las condiciones de la población en las áreas de influencia por parte de los empresarios, quienes generan incrementos del $30 \%$ en los ingresos de las familias campesinas, así como la formalización del empleo (afiliación al Sistema de Seguridad Social). En 2013 se generaron 24620 empleos directos, 49240 indirectos, 3824 industriales y 100832 en toda la cadena.

García y Calderón (2012) de FEDESARROLLO evalúan la mejora en las condiciones de vida en términos del desarrollo rural y los efectos de la implementación de la política de biocom- 
bustibles. Estos autores estimaron una generación de empleos directos para 2007 de 32052 y 6643 en actividades industriales. Debido a las reasignaciones de hectáreas destinadas a la producción de caña de azúcar, originalmente orientadas a la producción de azúcar para exportación, y ahora orientadas hacia producción etanol, el estudio concluye que no se generaron nuevos empleos, pues se considera que la recolección de la cosecha pasó de ejecutarse de manera mecanizada mediante tractores y no usando mano de obra local. Lombana et al. (2015) mencionan las bondades en la generación de empleo rural, pero no las cuantifican. El Consejo Nacional de Política Económica y Social (CONPES) (2009) analiza el sector de palma de aceite, indicando la generación de 145 000 empleos directos e indirectos, pero no discrimina los empleos del sector de biocombustibles, aunque se indica que el cálculo se realiza a razón de un empleo directo por cada 8 ha, y 1,5 empleos indirectos por cada directo. El DANE (2016), en convenio con FEDEPALMA, realizó la Encuesta Nacional de Empleo Directo en el sector palmero. Este estudio, si bien analiza el nivel de empleo en el sector, no separa específicamente para la generación de biocombustibles, por lo que no se incorporó en el presente estudio.

Otros trabajos para Colombia utilizaron enfoques desde la macroeconomía para evaluar la política, utilizando modelos de equilibrio general o de análisis de política comercial y arancelaria, pero sin enfocarse en la medición de la generación de empleo (Arbeláez et al., 2010; Corredor, 2017; Delgado et al., 2015; Manrique et al., 2008; Valencia \& Cardona, 2014). También evidenciamos trabajos similares al aquí propuesto para otros países del mundo, que revisan el impacto de la promoción de biocombustibles sobre el empleo (Neuwahl et al., 2008; Silalertruksa et al., 2012), aunque ninguno se centró en las particularidades del caso colombiano.

\section{DATOS ESTADÍSTICOS}

En la primera parte se analiza el comportamiento de las variables de interés, remarcando los principales hechos que han afectado su evolución en el tiempo, tales como modificación de políticas, choques climáticos, ampliación de la capacidad instalada en el sector de biocombustibles, entre otros. En la segunda parte de esta sección, se lleva a cabo la estrategia empírica del trabajo con un análisis de estacionariedad de las series y la formulación de los modelos VAR; luego se hace un contraste de longitud de retardo y se formalizan las funciones impulso respuesta de los respectivos modelos.

A partir del informe de FEDESARROLLO titulado Evaluación de la política de biocombustibles en Colombia (2012), y del informe de APEC titulado $A$ Study of Employment Opportunities from Production in APEC Economies (2010b), para cada sector de biocombustibles encontramos lo siguiente: 


\section{Etanol}

El proceso de producción de etanol genera empleo tanto no calificado como calificado, en el ámbito agrícola e industrial. Sin embargo, el principal impacto es el sector primario (cultivo de caña). La cantidad y el tipo de mano de obra que se requieren, varían de manera significativa dependiendo del nivel de mecanización en los cultivos de caña de azúcar. En los cultivos no mecanizados, la intensidad de mano de obra es mayor por las labores de corte, que emplea en su mayoría trabajo no calificado. Los cultivos mecanizados, por su parte, son menos intensivos en mano de obra y requieren trabajadores con cierto grado de conocimientos técnicos para operar la maquinaria. Según FEDESARROLLO (2012), los empleos directos generados por hectárea son de 0,235 para el cultivo no mecanizado y de 0,028 para el mecanizado.

Según cálculos de FEDESARROLLO (2012), para la cosecha manual se requieren aproximadamente 40 personas por hectárea durante 8 horas, mientras que el proceso mecanizado requiere en promedio 5 personas por hectárea durante 4 horas. Según el informe, para el 2012 en Colombia un $25 \%$ de los cultivos se encontraba mecanizado, porcentaje que ha aumentado con el desarrollo de producción de etanol como Bioenergy y Agrifuels, los cuales cuentan con un nivel de tecnificación del cultivo del $100 \%$. Se estima que los empleos generados en las destilerías son aproximadamente 60 por planta (FEDESARROLLO, 2012).

\section{Biodiésel}

Se calcula que el sector palmero emplea en promedio un trabajador por cada 3,2 hectáreas cultivadas, o lo que es equivalente 0,31 empleos por hectárea (FEDESARROLLO, 2012). Según se describe en el documento de referencia, el efecto de incremento en la producción de biodiésel es positivo en términos de empleo, si se tiene en cuenta que las plantaciones de palma se ubican en su mayoría en zonas que se dedicaban a la ganadería extensiva, que es poco intensiva en mano de obra (200 ha/trabajador).

La Gerencia Privada PTP (2012) hace sus estimaciones de empleo generados por el sector del biodiésel y, como se muestra en la evolución de los datos históricos Tabla 1, se encuentra que para 2011 el cultivo de palma dedicado al sector del biodiésel generó 21853 empleos directos; 43000 empleos indirectos y 80000 personas beneficiadas por las actividades relacionadas con el cultivo, extracción y producción de biodiésel (citada por FEDESARROLLO, 2012). 
Tabla 1. Generación de empleo rural

\begin{tabular}{ccc}
\hline Año & $\begin{array}{c}\text { Generación de empleo rural } \\
\text { sector palmero (total) }\end{array}$ & $\begin{array}{c}\text { Empleo rural } \\
\text { para biodiésel }\end{array}$ \\
\hline 2007 & 38360 & 460 \\
\hline 2008 & 42119 & 2205 \\
\hline 2009 & 45067 & 8539 \\
\hline 2010 & 50461 & 19980 \\
\hline 2011 & 53421 & 21853 \\
\hline
\end{tabular}

Fuente: (Fedesarrollo, 2012).

Donde más se genera empleo de manera significativa es en la contratación de mano de obra agrícola. La cantidad de empleos que se crean en las plantas de transformación no es muy grande (60 por planta), incluyendo funciones operativas y administrativas (FEDESARROLLO, 2012). Con el fin de determinar el impacto potencial de la industria de los biocombustibles en las oportunidades de empleo en las economías de la APEC, la entidad construyó un modelo para capturar los costos de los insumos de operación de una planta de etanol o biodiésel y traducirlos en cifras de empleo. Estas cifras incluyen no solo las personas involucradas en la operación de la planta, sino también las que participan en el suministro de materia prima y su transporte. El estudio se basó en datos de las economías donde los biocombustibles han estado en producción durante muchos años (como Estados Unidos y Brasil-no miembro de la APEC-) (APEC, 2010b).

Se desarrolló un modelo de empleo para biocombustibles de primera generación, que permite estimar para cualquier país de interés el empleo permanente que se crearía por una determinada cantidad de producción de biocombustibles. Los derivados tenidos en cuenta fueron (1) etanol a partir del maíz; (2) etanol de caña de azúcar; (3) biodiésel a partir de aceite de palma y (4) biodiésel a partir de aceite de soya.

El estudio sugiere que los esfuerzos para desarrollar biocombustibles en las economías de APEC deben hacer hincapié en la creación de una economía basada en el conocimiento (KBE) y la modernización y mecanización de la agricultura. Empleos basados en el conocimiento que se centran en el suministro de productos y servicios sofisticados, tanto para uso en las operaciones de refinación como de obtención de materias primas. Los biocombustibles representan entonces una herramienta muy prometedora para el desarrollo de un sector agroindustrial basado en el conocimiento, gracias a la utilización de materias primas locales y al potencial para el procesamiento interno. 
Este aspecto de cualificar el trabajo es muy importante. En el estudio se considera que a medida que la industria de los biocombustibles se expande, los productos y servicios podrían ser vendidos en toda la región APEC y el mundo. En cuanto a los detalles del análisis, entregan las siguientes estimaciones:

- Etanol de maíz (usando Estados Unidos como el modelo de un "productor avanzado"). Aproximadamente 37000 empleos directos e indirectos están asociados con la producción de etanol de maíz de 34069 millones de litros anuales (MLy).

- El etanol de caña de azúcar (con Brasil como modelo). Cerca de 96 000 empleos directos e indirectos se crearían con el logro de una meta de producción de etanol de caña de azúcar de 5000 millones de galones por año (MGy).
- El biodiésel de aceite de palma (utilizando Malasia como modelo). Unos 41000 puestos de trabajo directos e indirectos serían creados en la consecución de un objetivo de producción de biodiésel de aceite de palma de 560 millones de litros anuales (MLy).

- Biodiésel de aceite de soya (con Estados Unidos como modelo). Cerca de 9500 puestos de trabajos directos e indirectos están asociados con la producción de biodiésel de 2650 millones de litros anuales (MLy) de aceite de soya.

La Tabla 2 muestra los diferentes cálculos del número de puestos de trabajo por millón de litros producido en un año, basados en modelos de biocombustibles de primera generación:

Tabla 2. Empleos por millón de litros por año para las principales materias primas usadas en la producción de biocombustibles

\begin{tabular}{ccccc}
\hline $\begin{array}{c}\text { Materia prima } \\
\text { Biocombustible }\end{array}$ & $\begin{array}{c}\text { Empleo } \\
\text { modelado } \\
\text { por } \\
\text { biorrefinería }\end{array}$ & $\begin{array}{c}\text { Tamaño asumido } \\
\text { de la biorrefinería }\end{array}$ & $\begin{array}{c}\text { Empleos por } \\
\text { millón de } \\
\text { galones por año }\end{array}$ & $\begin{array}{c}\text { Empleo } \\
\text { por millón } \\
\text { de litros } \\
\text { por año }\end{array}$ \\
\hline Etanol de maíz & 412 & $100 \mathrm{MGY}$ & 4.12 & 1.1 \\
\hline Etanol de caña de azúcar & 1920 & $100 \mathrm{MGY}$ & 19.2 & 5.1 \\
\hline Biodiesel de aceite de palma & 2930 & $40 \mathrm{MLY}$ & & 73.3 \\
\hline Biodiesel de aceite de soya & 316 & $90 \mathrm{MLY}$ & & 3.5 \\
\hline
\end{tabular}

Fuente: (APEC, 2010a)

Las cifras calculadas de empleos por millón de litros por año varían ampliamente, porque los modelos se derivan del análisis de diversos países productores. Una variabilidad significativa es de esperarse debido a la inclusión de materia prima diferencial, el grado de automatización/mecanización de las operaciones de refinería y de campo, y la ubicación (que afecta a los costos, 
la productividad y otros factores). Por ejemplo, el alto nivel de empleos para el biodiésel de aceite de palma (basado en el modelo de Malasia) es una reflexión sobre el gran número de trabajadores necesarios para la cosecha y el transporte de la fruta de palma, así como el significativo empleo generado en la extracción y operaciones de trituración. También podría reflejar un nivel más bajo de mecanización de la economía en general y del proceso de producción de biodiésel de aceite de palma en particular (APEC, 2010a).

El informe APEC utilizó diferentes modelos: en primer lugar, contempló el análisis input-output (I/O), el cual se puede utilizar para calcular los impactos indirectos sobre el empleo, el PIB, la renta y las exportaciones. Estos modelos consideran el tamaño de la superficie plantada y el precio de la materia prima (entre otras variables); en segundo lugar, los modelos de análisis social accounting matrix (SAM). Este análisis es una extensión del análisis input-output estándar y permite el cálculo de los impactos indirectos de la producción de biocombustibles sobre los ingresos del hogar; y en tercer lugar, se usaron modelos de equilibrio espacialmente explícitos: se trata de modelos que pueden ser utilizados para calcular el impacto de varios parámetros sobre la oferta y demanda de alimentos debido a la producción de biocombustibles.
En la Figura 1 puede observarse cómo la producción de biodiésel mantuvo una tendencia creciente desde 2009, el año posterior al inicio del programa de mezcla, hasta enero de 2015, con una producción máxima de 14,64 millones de galones en julio de 2013. Sin embargo, tal crecimiento se presentó de una manera más notoria entre los años 2009-2012, en parte, gracias a las medidas implementadas por el Ministerio de Minas y Energía para promover la producción del biodiésel, y a la entrada en funcionamiento de nuevas plantas productoras (DANE, 2016).

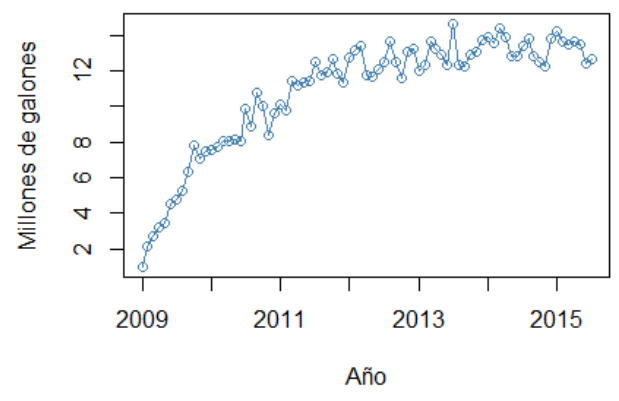

Figura 1. Producción de biodiésel

Fuente: cálculos de los autores con base en UPME (2019).

Dentro de las medidas implementadas se encuentran el documento CONPES 3510 de 2008, el cual contiene los lineamientos de política para promover la producción sostenible de biocombustibles en Colombia; el aumento en el porcentaje de mezclas decretado en el 2010 , pasando de un $5 \%$ a un $7 \%$ en Bogotá, el centro del país y los Llanos Orientales, y de un $8 \%$ a $10 \%$ en Santander, Antioquia y el occidente del 
país. Lo anterior motivado como respuesta al exceso de oferta que se tenía en el momento y a la entrada en operación de Eco-Diésel en el Magdalena Medio a partir del año 2010 (DNP, 2008).

Por otro lado, entre 2013 y 2015, el aumento de la capacidad de producción no fue muy dinámico y no se presentaron cambios importantes en las medidas que ya habían sido presentadas por parte del MME. También es importante resaltar en este análisis que el aceite de palma ha presentado una dinámica de crecimiento en las áreas sembradas entre los años analizados, lo cual permite que la producción del biocombustible pueda expandirse como respuesta a estímulos a la oferta.

En suma, el aumento de la capacidad instalada y las disposiciones del Ministerio de Minas y Energía influyeron para que la producción de biodiésel creciera notablemente entre los años 2009-2012, a diferencia de los años 2013-2015, cuando no se presentaron cambios normativos relevantes y la capacidad instalada no presentó un aumento significativo, a excepción del año 2013, en el cual se generó la mayor producción del biocombustible gracias a la entrada en operación de Biocastilla de Castilla La Nueva y Romil de Colombia (Ministerio de Minas y Energía, 2009).

En la Figura 2 es posible observar como la producción de etanol ha presentado una tendencia de crecimiento durante los años 2009 y 2015, con una serie de fluctuaciones que son propias de las dinámicas del cultivo de la caña de azúcar (principal materia prima del etanol). El mínimo histórico se registra a finales del año 2010 con un nivel de producción de 3,56 millones de galones de etanol, consecuencia de la baja cosecha de caña de azúcar debida al fenómeno de La Niña que, incluso, llevó a la suspensión del programa de oxigenación en todo el país para diciembre de 2010. Ya para el 2011 se evidencia una recuperación del sector y un crecimiento en la producción de etanol hacia un nivel más elevado de lo que se venía presentando antes del fenómeno climático, en parte, gracias a las inversiones destinadas a la ampliación de 3 de las 5 destilerías en funcionamiento, lo que llevó la capacidad instalada de $1,05 \mathrm{~m} / \mathrm{lt} /$ día a $1,25 \mathrm{~m} /$ 1t/día. De igual forma, en los años subsiguientes al 2011 la producción de etanol continuó con una tendencia al alza, en concordancia con la plena operación de las destilerías existentes y con la fijación del porcentaje de mezclas en un $10 \%$.

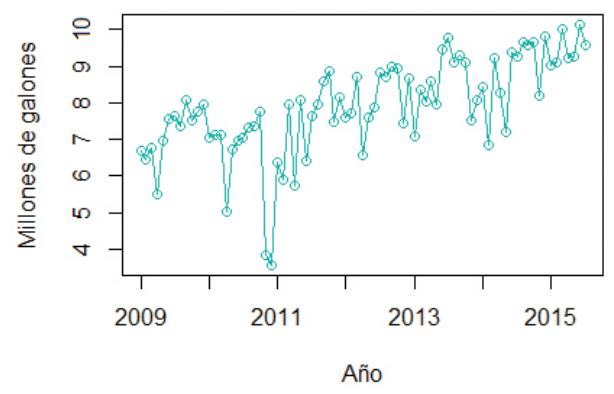

Figura 2. Producción de etanol

Fuente: cálculos de los autores con base en UPME (2019). 


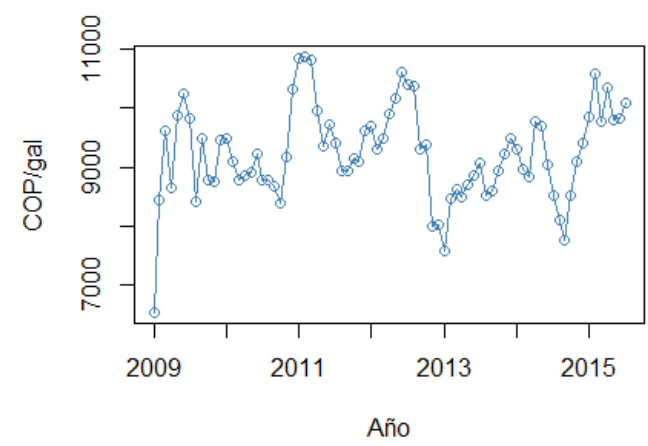

Figura 3. Precios constantes del biodiésel

Fuente: cálculos de los autores con base en FEDEBIOCOMBUSTIBLES (2019).

Al iniciar el análisis del comportamiento de la serie, se puede observar cómo a inicios del año 2009 se presentan unos niveles bajos en el precio del biodiésel en comparación con el resto de los años analizados, y esto ocurrió básicamente debido a los descensos en el precio internacional de la palma de aceite, con una disminución en el precio del $61 \%$ entre marzo y noviembre de 2008. Estos precios del biocombustible empiezan a tener una recuperación a partir de marzo de 2009 y mantienen cierta estabilidad hasta noviembre de 2010 con un precio promedio aproximado de 9100 pesos por galón; sin embargo, para diciembre de 2010 los precios en los mercados internacionales de la palma de aceite sufren una fuerte alza hasta alcanzar un nivel de 10300 pesos por galón, continuando con una leve fluctuación hasta finales del año 2012, cuando se presenta una tendencia a la baja de los precios, debida al dinamismo de producción de aceite de palma y soya en el Sudeste
Asiático. Para noviembre de 2012, el Ministerio de Minas y Energía, mediante Resolución 91664, aumentó el porcentaje de mezclas de biocombustibles para uso en motores diésel a un $8 \%$ en la ciudad de Bogotá, el centro del país y los Llanos Orientales.

Vale la pena destacar que tanto los porcentajes de mezclas como los precios del biodiésel son regulados por el Ministerio de Minas, el cual, según el documento CONPES 3510 de 2008, estableció un esquema regulatorio en los precios que busca reconocer los costos de oportunidad de los productos complementarios y de los usos alternativos de la materia prima utilizada en su producción, así como los costos eficientes de transformación de las materias primas. Además, al biodiésel se le han dado una serie de incentivos tributarios que buscan, por un lado, volver competitivo este biocombustible y, por otro, reducir los precios del diésel que rigen en Colombia. Dichos incentivos son: (1) incentivo de exención de impuesto de renta por cultivos de tardío rendimiento; (2) exención de IVA para el biocombustible destinado para mezcla con combustibles fósiles; (3) exención de la sobretasa global al combustible a los biocombustibles destinados para mezcla con combustibles fósiles; (4) zonas francas agroindustriales.

Lo anterior pone de manifiesto de qué manera los biocombustibles producidos en Colombia son subsidiados gracias a las reglamentaciones dictadas por el 
Gobierno con el objetivo de incentivar el desarrollo de los biocombustibles en el ámbito nacional. Tanto el marco regulatorio, determinado por el MME, como los incentivos tributarios, también se cumplen para el caso del etanol y sus precios, los cuales se fijan el primer día de cada mes y rigen hasta el último día del mes. A continuación, veremos el comportamiento de dichos precios entre los años 2009 y 2015.

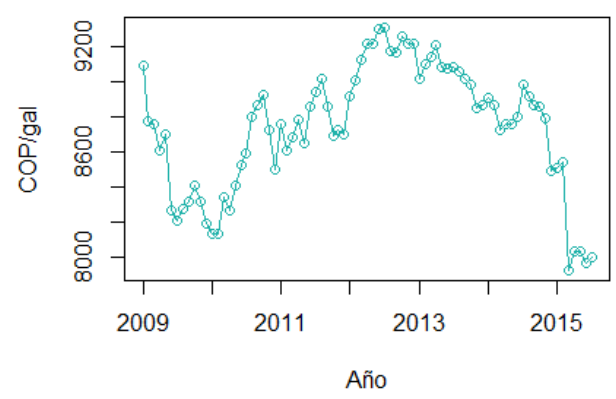

Figura 4. Precios constantes del E8.

Fuente: cálculos de los autores con base en Fedebiocombustibles (2019).

Como se puede observar en la Figura 4, entre el año 2009 y 2010 hubo un descenso en los precios de la gasolina mezclada con etanol al $8 \%$. Situación que es ocasionada, primero, por los cambios en la reglamentación del porcentaje de mezclas que se hicieron en estos años como consecuencia de insuficiencia en la oferta, que llevaron a un descenso del $10 \%$ al $8 \%$ en la mezcla; segundo, debido al cambio en la estructura y determinación de los precios del etanol.

Luego, entre los años 2011 y 2013, un aumento en el nivel de precios de este combustible mezclado se explica por los máximos históricos del precio internacional del azúcar (2011), que impactaron el precio del etanol; por otro lado, los significativos aumentos en el precio internacional del petróleo condujeron al aumento del precio de la gasolina en este mismo período.

Entre los años 2013 y 2015 es posible percibir la dinámica de caída en el nivel de precios, lo cual se justifica, como se aprecia en Figura 5, en que se permite la importación de etanol para satisfacer el mercado interno, con una fuerte tendencia hasta mediados del 2014. Estas importaciones dan paso a nueva competencia con precios más bajos que erosionan aún más el precio del etanol. Se puede observar además que la tendencia decreciente en los precios es más pronunciada a partir de finales del año 2014 y comienzos del 2015, principalmente a causa de la fuerte caída de los precios internacionales del petróleo.

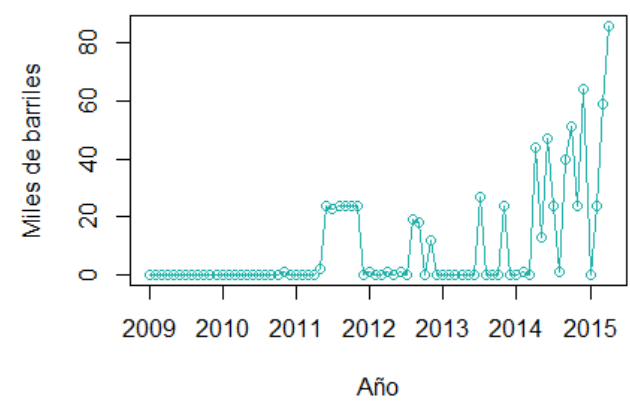

Figura 5. Importaciones de etanol desde Estados Unidos

Fuente: cálculos de los autores con base en EIA (2019). 
Con la intención de ilustrar acerca de la dinámica de las importaciones de etanol por parte de Colombia, es importante tener en cuenta que Estados Unidos es nuestro principal socio comercial, a su vez que es el principal productor de etanol en el mundo; por tanto, es pertinente observar cómo se ha dado este proceso de importaciones de etanol desde dicho país.

Para hacer un análisis apropiado de la serie de empleo en la fabricación de azúcar y panela es necesario tener en cuenta que a finales de 2008 y durante un promedio de 56 días, un grupo de corteros de caña bloqueó la producción de ocho ingenios, que representaban el $68 \%$ de la molienda de caña, lo cual imposibilitó la cosecha, trasladando buena parte de ella al año 2009. Los efectos negativos del bloqueo fueron evidentes en la economía de la región, en especial para el empleo e ingreso de miles de colaboradores de diversas áreas de los ingenios, a quienes se les impidió la entrada a sus puestos de trabajo (Asocaña, 2010).

La situación anterior se refleja en la tendencia decreciente hacia 2009, como puede apreciarse en la Figura 6. Con respecto al año 2010, se empieza a ver una recuperación de dicho índice debido al crecimiento del sector azucarero registrado durante los primeros meses del año, y a la acumulación de cosechas presentadas para finales del año. De igual forma, las condiciones climáticas adversas que se dieron al final del 2010 ocasionaron reducciones en la cosecha de la caña de azúcar, lo cual se vio reflejado en la disminución del índice durante los primeros meses de 2011; sin embargo, estos efectos se fueron superando gradualmente, también gracias a que para el segundo semestre del año 2011 se hicieron efectivas una serie de inversiones que permitieron la ampliación en 200000 litros de etanol en las destilerías de los ingenios Mayagüez, Providencia e Incauca.

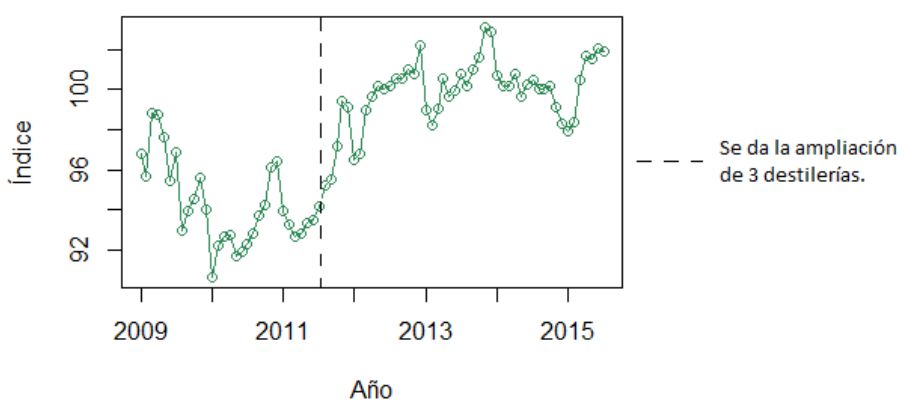

Figura 6. Índice de empleo de la fabricación de azúcar y panela (EMM)

Fuente: cálculos de los autores con base en DANE (2019). 
Para finalizar, se puede observar en la Figura 6 que a partir del año 2012 el índice de empleo se mantiene estable relativamente y con algunas fluctuaciones no muy marcadas, que se deben propiamente al comportamiento del sector azucarero, el cual depende, entre otras cosas, de los precios internacionales del azúcar, la demanda interna de los diferentes productos derivados de la caña de azúcar, del área sembrada de caña y de las condiciones climáticas. En la Figura 7 se pueden evidenciar los cambios presentados en la generación de empleo para el sector de fabricación de sustancias y productos químicos, código CIIU correspondiente a la fabricación de biodiésel. En el período analizado el empleo no se sostiene como lo hacen los niveles de producción en Figura 1, sino que incluso se percibe un descenso desde 2012, al parecer por sustitución de mano de obra en la recolección y procesamiento por maquinaria y equipo.

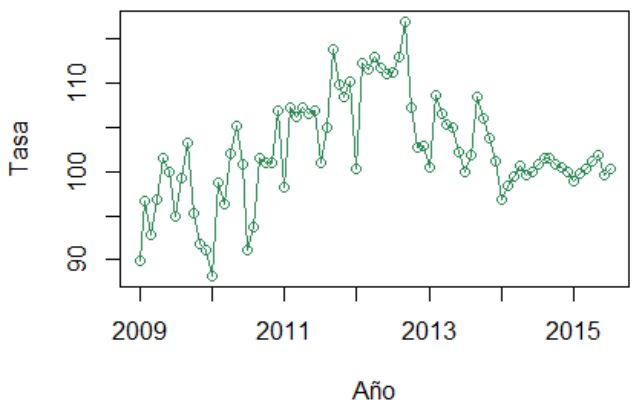

Figura 7. Índice de empleo en la fabricación de sustancias y productos químicos (EMM)

Fuente: cálculos de los autores con base en DANE (2019).
En la Tabla 3 se presenta la información estadística empleada en la aplicación de la metodología empírica. Se incluyen 79 observaciones mensuales tanto de variables endógenas como exógenas. Por su parte, tanto la producción de biodiésel como la producción de etanol se encuentran medidas en galones, y la fuente consultada para ello fue el Sistema de Información de Petróleo y Gas (SIPG); el precio del biodiésel y de la gasolina con mezclas de etanol del $8 \%$ (E8) se encuentran en pesos constantes indexados por el IPP con base en el año 2014, tomando como fuente la información suministrada en la página de FEDEBIOCOMBUSTIBLES; la producción de azúcar se mide en toneladas y la fuente consultada fue ASOCAÑA (2016); la producción de aceite de palma se mide en toneladas y la información se encuentra en el Sistema de Información Estadística del Sector Palmero (SISPA); por último, el índice de empleo en la fabricación de sustancias químicas básicas, y sus productos (en donde se incluye el empleo del biodiésel) se encuentra referenciado en la versión 4 del código CIIU con el catálogo 2010 de la Encuesta Mensual Manufacturera (EMM) elaborada por el Departamento Nacional de Estadística (DANE), teniendo en cuenta que a partir del 2014 se desestacionaliza la serie; y el índice de empleo en la elaboración de azúcar y panela se encuentra referenciado en el código CIIU 1070 de la EMM (DANE, 2019). 
Tabla 3. Estadísticas descriptivas

\begin{tabular}{lcccc}
\hline \multicolumn{1}{c}{ Variable } & Media & Desv. Std. & Mínimo & Máximo \\
\hline Producción biodiésel & 10863702 & 3241043 & 991167 & 14645024 \\
\hline Precio biodiésel & 9236 & 7937 & 6529 & 10875 \\
\hline Empleo fabricación de sustancias químicas & 102,35 & 59,08 & 88,17 & 116,98 \\
\hline Producción de aceite & 81003 & 17639 & 50444 & 125952 \\
\hline Producción etanol & 7896672 & 1313790 & 3564384 & 10134244 \\
\hline Precio del E8 & 8745 & 357 & 7929 & 9309 \\
\hline Empleo elaboración de azúcar y panela & 97,68 & 33,02 & 90,66 & 103,12 \\
\hline Producción de azúcar & 184353 & 40186 & 80371 & 263434 \\
\hline
\end{tabular}

Fuente: cálculos propios.

\section{METODOLOGÍA}

\section{Estacionariedad de las series}

La prueba de Cavaliere y Georgiev (2006) se basa en el siguiente modelo:

$$
\begin{gathered}
X_{t}=\varphi^{\prime} Z_{t}+Y_{t}+\mu_{t} \\
Y_{t}=\alpha Y_{t-1}+ \\
u_{t}=\sum_{i=1}^{p} \quad \gamma_{i} u_{t-1}+\varepsilon_{i}
\end{gathered}
$$

Donde $X_{t}$ es la variable observable, $Y_{t}$ es un proceso autorregresivo no observable, $Z_{t}$ es un vector $p \times 1 \mathrm{de}$ términos determinísticos conocidos (constante $\mathrm{y} / \mathrm{o}$ tendencia), $\varphi$ es un vector fijo conformable con $Z_{t}$ y $\mu_{t}$ representa el componente no observable de cambios de nivel. De $u_{t}$ se supone que es estable. $\hat{\gamma}$ y $\hat{\alpha}$ son estimado mediante OLS para $\gamma_{i}$ y $\alpha$. El modelo se basa en dos supuestos: primero, las raíces del polinomio $\Gamma(z)=1-\sum_{i=1}^{k}$ $\gamma_{i} z^{i}$ tienen módulos mayores que $1, \mathrm{y}$ segundo, $\varepsilon_{t}$ distribuye independiente e idénticamente distribuido con media cero y varianza positiva (Cavaliere \& Georgiev, 2006). Además, se considera que la componente de cambio de nivel se encuentra especificada por la ecuación [4], donde $\delta_{s}$ es una variable dummy que solo toma el valor de 1 , si en el momento $t$ ocurre un cambio de nivel. Mientras $\theta_{S}$ representa el cambio en dicho periodo $t$.

$$
\mu_{t}=\sum_{s=1}^{t} \quad \delta_{s} \theta_{s}
$$

Castaño y Sierra (2012) presentan el procedimiento sugerido por Cavaliere y Georgiev (2006) en los siguientes casos:
i. $\varphi$ y p es conocido
ii. $\varphi$ y $\mathrm{p}$ es conocido
iii. $p$ es desconocido 
Para el primer caso, se realiza una regresión de $\Delta X_{t}$ sobre las variables dummy de cambio de nivel, se debe incorporar una variable dummy por cada cambio. El estimador de $\mu_{t}$ es:

$$
\widehat{\mu_{t}}=\sum_{s=1}^{t} \quad \delta_{s} \Delta X_{t}
$$

Cuando no se conoce la ubicación de los diferentes cambios de nivel, es decir $\delta_{s}$ es desconocido, Castaño y Sierra (2012), citando a Cavaliere y Georgiev (2006), sugieren utilizar una estimación de , la cual se puede obtener realizando el proceso de detección de observaciones atípicas en series de tiempo de Chen y Tiao (1990) o de Chen y Liu (1993). Cavaliere y Georgiev (2006) muestran que la prueba $\mathrm{ADF}$ no se ve influenciada por tomar dicha estimación. Para obtener la serie ajustada (dejumped), se resta la componente anterior del cambio de nivel a la variable observada:

$$
X_{t}^{\delta}=X_{t}-\widehat{\mu_{t}}
$$

Finalmente, se procede con la prueba tradicional ADF sobre la serie dejumped $X_{t}^{\delta}$. Cuando se presenta el segundo caso ( $\varphi \neq 0$ y p es conocido), de nuevo se obtiene la serie dejumped removiendo los cambios de nivel de la serie observada, para luego aplicar el procedimiento de una seudo eliminación de tendencia GLS propuesto por Elliot et al. (1996) a la serie ajustada que se obtuvo. Y final- mente, empleando el software STATA se realiza la prueba $\mathrm{ADF}$ sobre la serie sin tendencia (Castaño \& Sierra, 2012).

El tercer caso, cuando el rezago $p$ de la ecuación [3] es desconocido, se define inicialmente $p_{\text {máx }}=12 *\left(\frac{N}{100}\right)^{0.25}$, y se procede a encontrar los cambios de nivel cuando estos no son conocidos; para posteriormente obtener la serie ajustada o dejumped. A partir de la serie ajustada se emplea un criterio estándar para estimar $p$, y con dicha estimación se realiza la prueba ADF. Proponemos un modelo con cuando $\varphi=0$ y $\mathrm{p}$ es desconocido:

$$
\begin{gathered}
X_{t}=\varphi_{0}+Y_{t}+\mu_{t} \\
Y_{t}=\alpha Y_{t-1}+u_{t} \\
u_{t}=\sum_{i=1}^{p} \quad \gamma_{i} u_{t-1}+\varepsilon_{i} .
\end{gathered}
$$

\section{Vectores autorregresivos}

De manera general, un modelo vectorial autorregresivo se puede escribir como:

$$
\begin{aligned}
Y_{t}= & c+\Phi_{1} Y_{t-1}+\Phi_{2} Y_{t-2} \\
& +\cdots+\Phi_{p} Y_{t-P}+\varepsilon_{t}
\end{aligned}
$$

La inclusión de más variables y más retardos en el modelo, hacen la estimación poco eficaz en términos de significancia de los parámetros, pues la evidencia sugiere que hasta doce retardos es lo apropiado para modelar series de corte 
mensual. Pero, por otro lado, si el número de parámetros excede el número de observaciones, entonces la especificación estaría sobreidentificada y no sería posible elaborar la estimación. Es importante destacar que si el análisis preliminar de la información estadística (como en la sección anterior 3.A) arroja evidencia definitiva de que las series no son estacionarias, el procedimiento habitual sugerido en la práctica es buscar relaciones a largo plazo entre algunas (o todas) las variables, lo que lleva necesariamente a la noción de cointegración.

Una importante característica de los modelos VAR es la posibilidad de calcular el efecto de un choque positivo de una variable sobre otra, comúnmente conocida como la función impulso respuesta-IRF-:

$$
\frac{\partial y_{t+s}}{\partial \varepsilon_{t}}=\Psi_{s}
$$

donde $\Psi_{S}$ es una matriz $n \times n$, siempre que el VAR sea estacionario y pueda expresarse como un proceso vectorial de media móvil, y la interpretación derivada de la ecuación (11) es que el elemento $i, j$ de la matriz $\Psi_{s}$ identifica las consecuencias de una variación en el momento $t$ de la variable $y_{i}$ para los valores de la variable $y_{j}$ en $t+s$, esto es, para $y_{j, t+s}$ . Así, estas funciones representan el impacto de las variables (retardos propios y retardos de las demás variables) sobre las variables independientes.
Técnicamente hablando, un choque en una variable tiene un efecto en cadena que se expande a las demás variables, de modo que una vez estimado el VAR, lo que es posible calcular o simular son los choques en la producción de biocombustibles y su efecto sobre el empleo $E_{t}$ en $s>0$ periodos adelante, es decir:

$$
\frac{\partial \ln \ln E_{t+s}}{\partial \varepsilon_{t}}=\Psi_{s}=\frac{\partial \ln \ln E_{t+s}}{\partial \ln \ln X_{t}} .
$$

El efecto en cadena recogido por la expresión [12] representa la elasticidad $X_{t}$ y empleo de corto o mediano plazo que, aunque es móvil, prevalece en $t=1,2,3, \ldots ., s$. Donde $X_{t}$ es cualquiera de las variables a la que se le produce un choque positivo (normalmente medido en desviaciones estándar), como por ejemplo la producción de etanol o biodiésel, los precios, etc. Así, el análisis de series de tiempo multivariadas es un instrumento riguroso para modelar variables que están correlacionadas en el tiempo, a diferencia de los modelos univariados que no permiten dicho análisis.

\section{RESULTADOS}

Para la estimación se formularon dos modelos VAR; uno asociado a la industria del biodiésel, y el otro asociado a la industria del etanol. En el primero, las variables que se incluyen son: índice de empleo en la fabricación de otros productos químicos, nivel de producción del biodiésel, precio del biodiésel y la producción de aceite. Para el segundo modelo se utilizan las siguientes varia- 
bles: índice de empleo en la fabricación de azúcar y panela, la producción de etanol, el precio de la gasolina con una mezcla del $8 \%$ de etanol (E8) y el nivel de producción de azúcar.

Para estimar los modelos VAR fue necesario, en primer lugar, establecer si las series individualmente son estacionarias en covarianza o si están integradas. Para este propósito se realizan procedimientos de contraste de Cavaliere y Gioergiev (2008), dado que los contrastes habituales de Dickey-Fuller o el de Phillip-Perron no son confiables cuando las series, como en este caso, presentan cambios de nivel.

Sin embargo, antes de realizar cualquier tipo de prueba de raíz unitaria se tiene que garantizar que las series son de varianza estable. Al hacer un análisis gráfico se observó como la mayoría de las series seleccionadas para el modelo ven afectada su varianza a medida que el nivel de la serie cambia; por tanto, recurrimos a la transformación de Box-Cox (1996) para tratar de estabilizar la varianza. El resultado subsecuente nos arroja que la transformación de las series en logaritmos permite la suavización de las series a medida que va evolucionando en el tiempo, lo cual también nos permite interpretar los coeficientes asociados al VAR como elasticidades. Ahora sí se procede a las pruebas de raíz unitaria.

\section{Estacionariedad}

En los contrastes llevados a cabo, se admite rechazar la hipótesis de raíz unitaria como máximo al $10 \%$ de significancia para todas las series en logaritmos, excepto para la serie del precio del E8. Es importante destacar que la información no arroja evidencia de cointegración (relaciones cointegrantes o de equilibrio a largo plazo) entre las variables estudiadas.

La razón para que inicialmente la serie en logaritmos del precio del E8 concluya a favor de la no estacionariedad por medio de las dos pruebas de raíz unitaria utilizadas en esta sección. Después de hacer un análisis de identificación de outliers, se concluye que la serie del precio del E8 presenta cambios de nivel en junio de 2009, agosto de 2010 y marzo de 2015; dichos cambios de nivel estaban causando la no estacionariedad de la serie. Por ello procedimos a darle el adecuado tratamiento a la serie para marginar la distorsión derivada del cambio de nivel; este consistió en la aplicación de la prueba de Cavaliere y Georgiev (2006) disponible en la Tabla 4, la cual propone formar un dejumped a la serie original, generando las variables dummie correspondientes a los períodos en que se presentó tal cambio de nivel. 
Tabla 4. Test Cavaliere y Giorgiev (2006)

\begin{tabular}{ccccc}
\hline & \multicolumn{2}{c}{ PP (default Lags) } & \multicolumn{2}{c}{ ADF (lags 3) } \\
\hline Variable & $\mathbf{Z ( t )}$ MacKinnon & Conclusión & $\mathbf{Z ( t )}$ MacKinnon & Conclusión \\
& & & & \\
\hline Producción. Biodiésel & 0,0000 & Estacionario & 0,0000 & Estacionario \\
\hline Producción. Biodiésel & 0,0002 & Estacionario & 0,0002 & Estacionario \\
\hline Producción. Aceite & 0,0000 & Estacionario & 0,0000 & Estacionario \\
\hline Empleo. Sec. Quim & 0,0000 & Estacionario & 0,0000 & Estacionario \\
\hline Producción. Etanol & 0,0001 & Estacionario & 0,0001 & Estacionario \\
\hline Producción. Azúcar & 0,0000 & Estacionario & 0,0000 & Estacionario \\
\hline Empleo Sec. Azúcar & 0,0000 & Estacionario & 0,0000 & Estacionario \\
\hline Pe8. Cdj & 0,0023 & Estacionario & 0,0015 & Estacionario \\
\hline
\end{tabular}

Fuente: cálculos propios.

Posteriormente, sobre la serie transformada en la dejumped, se aplicó la prueba aumentada de Dickey y Fuller de raíz unitaria y se comprobó que dicho tratamiento produce una serie estacionaria en niveles.

\section{Estimación del VAR, funciones im- pulso-respuesta y proyecciones}

A efectos de evaluar la respuesta generalizada de un choque en diversas variables que afectan el empleo, se presentan los resultados de las funciones impulso-respuesta tanto del sector de producción de etanol como de biodiésel. El efecto se calcula para 12 y 24 meses adelante (solo reportamos la de 24 meses). En las figuras 8 y 9 se muestran las simulaciones de un choque positivo, exactamente de una desviación estándar, sobre el nivel de producción de biodiésel y/o etanol, en nivel de producción de azúcar y etanol, así como un choque de la misma magnitud en niveles de precios de biodiésel, niveles de producción de aceite de palma y biodiésel. Los resultados indican que modificar la normativa de mezcla obligatoria llevaría a un incremento del empleo que, en apariencia, es transitorio. Sin embargo, su efecto no es muy apreciable y, por el contrario, se diluye rápidamente en el tiempo. Esto es consistente con la metodología empleada, en la cual todas las variables son estacionarias con cambio de nivel, y por lo tanto, las correlaciones dinámicas proceden de un proceso vectorial estable, es decir, de vectores autorregresivos estacionarios en covarianza. 

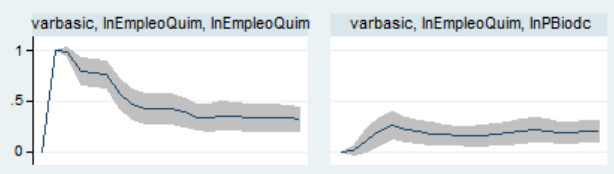

varbasic, InEmpleoQuim, InProdAceite

varbasic, InEmpleoQuim, InProdBiod

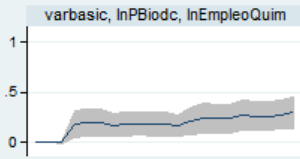

varbasic, InPBiodc, InPBiodc
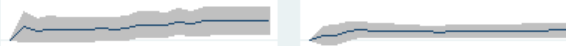

varbasic, InPBiodc, InProdAceite

varbasic, InPBiodc, InProdBiod
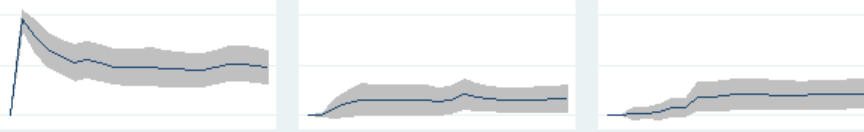

varbasic, InProdAceite, InEmpleoQuim

varbasic, InProdAceite, InPBiodc

varbasic, InProdAceite, InProdAceite

varbasic, InProdAceite, InProdBiod
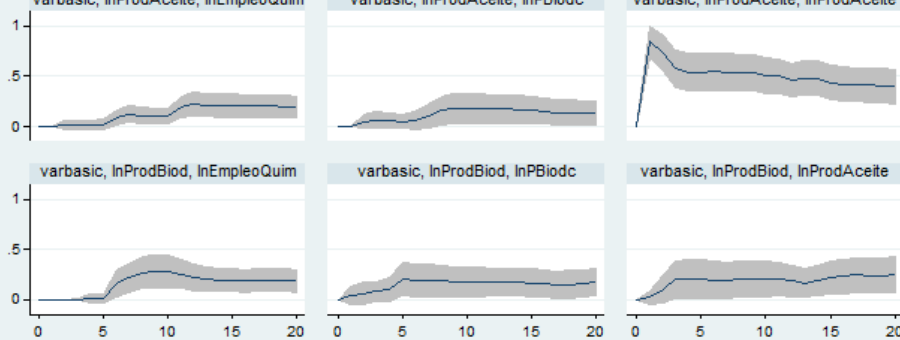

varbasic, InProdBiod, InPBiodc

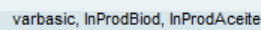

varbasic, InProdBiod, InProdBiod
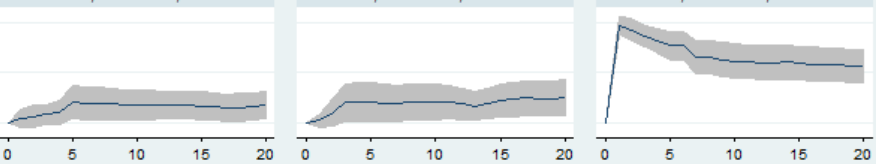

step

\section{$95 \% \mathrm{Cl}$}

\section{fraction of mse due to impulse}

Graphs by irfname, impulse variable, and response variable

Figura 8. Resultados de la función impulso-respuesta para el sector biodiésel

Fuente: cálculos propios. 


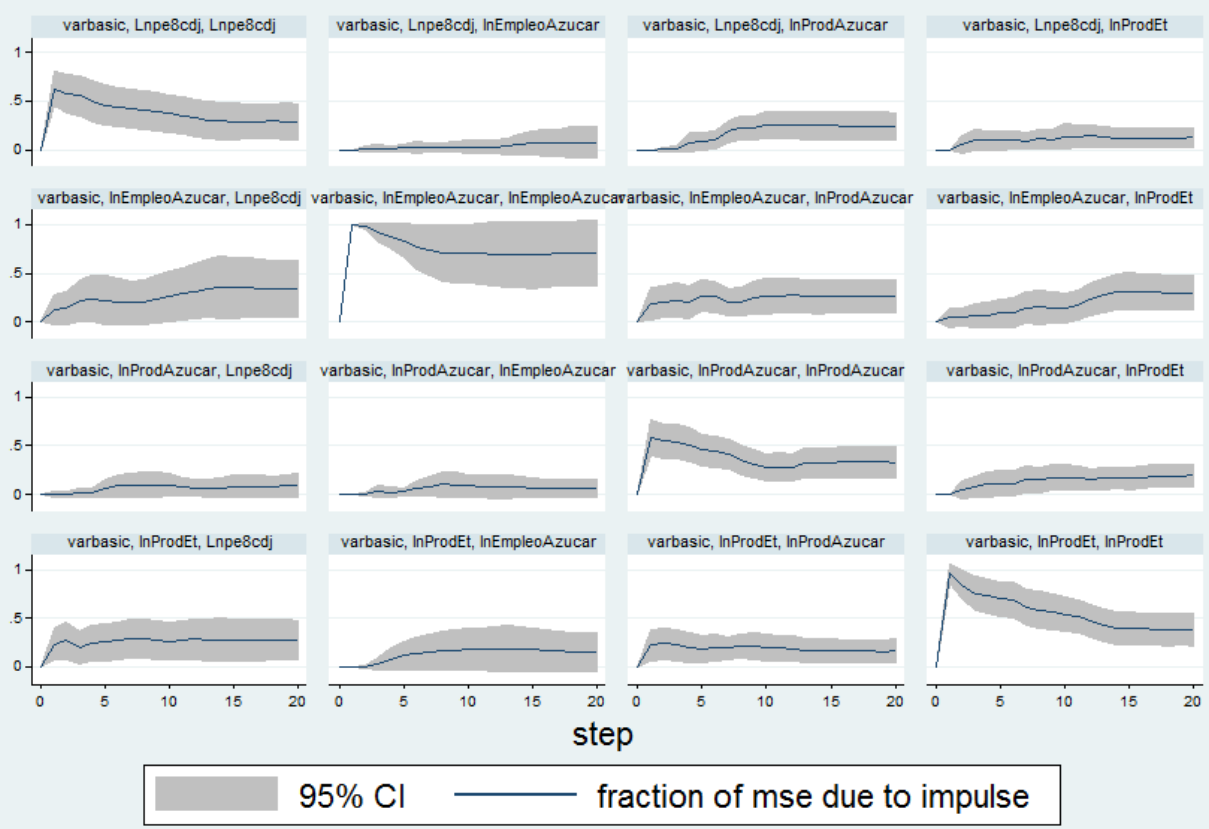

Graphs by irfname, impulse variable, and response variable

Figura 9. Resultados de la función impulso-respuesta para el sector de producción de etanol

Fuente: cálculos propios.

\section{CONCLUSIONES}

En este trabajo se analiza el posible efecto que tiene la aplicación de la política nacional de biocombustibles sobre la generación de empleo rural en Colombia. Analizamos su proceso de implementación a partir de la modificación de la regulación del sector y eventos exógenos relevantes entre los años 2009 a 2015, tales como cambios regulatorios y fenómenos climáticos atípicos, es decir, que no corresponden a decisiones óptimas de oferta o demanda de los agentes. Se construyó una base de datos que a través de un modelo de VAR per- mite diagnosticar la hipótesis defendida tanto por el Gobierno nacional como por muchos productores que abogan no sólo por sostener sino ampliar el alcance de la política, soportando su posición en que el sector es un generador de empleo, en especial en zonas rurales.

Los resultados estimados en el modelo permiten identificar que un choque positivo en cualquiera de las variables de interés muestra un efecto transitorio y no muy fuerte sobre el empleo en el sector. El artículo entonces plantea una discusión de política sobre la per- 
tinencia de la estrategia de promover y profundizar las mezclas de combustible fósil con biocombustibles. La discusión, quizá, puede redirigir al cambio de los objetivos de promover las mezclas, como el medioambiente, la disminución de material particulado y la seguridad energética, pero no el de la generación de empleo rural no calificado, cortadores y recolectores de materia prima (caña y aceite de palma) principalmente.

\section{AGRADECIMIENTOS}

Este artículo se desarrolló dentro de la Convocatoria de Investigación Programática 2016 Área de Ciencias Sociales, Humanidades y Artes de la Universidad de Antioquia. Proyecto CODI n. ${ }^{\circ}$ 2016-13242, titulado "Producción de biocombustibles y ge- neración de empleo rural en Colombia, un análisis mediante vectores autorregresivos VAR".

Los autores agradecemos los comentarios de los asistentes al Seminario de Economía de la Facultad de Economía de la Universidad Autónoma Latinoamericana (UNAULA) y de los evaluadores anónimos de la Revista Apuntes del Cenes. Agradecemos también la colaboración en la elaboración de este documento al joven investigador Santiago Palacio Ciro, economista de la Universidad de Antioquia, quien participó como estudiante en formación en el proyecto.

\section{DECLARACIÓN DE CONFLCITO DE INTERES}

Los autores declaran que no existe conflicto de intereses. 


\section{REFERENCIAS}

APEC. (2010a). A Study of Employment Opportunities from Biofuel Production in APEC Economies. APEC Energy Working Group. Ottawa.

APEC. (2010b). Biofuel Costs, Technologies and Economics in APEC Economies. Delaware. www.apec.org

Arbeláez, M. A., Estacio, A. \& Olivera, M. (2010). Impacto socioeconómico del sector azucarero colombiano en la economía nacional y regional. Fedesarrollo. https://www.repository.fedesarrollo.org.co/handle/11445/168

ASOCAÑA. (2010). Informe anual Asocaña 2010-2011. Asocaña.

ASOCAÑA. (2016). Aspectos generales del sector azucarero colombiano 20152016. Informe Anual 2016. Asocaña.

Barrientos-Marin, J., Tabares-Orozco, E. \& Velilla, E. (2018). Forecasting Electricity Price in Colombia: A Comparison between Neural Network, Arma Process and Hybrid Models. International Journal of Energy Economics and Policy, 8(3), 97-106. https://www.econjournals.com/index.php/ijeep/article/view/6181

Castaño, E. \& Sierra, J. (2012). Sobre la existencia de una raíz unitaria en la serie de tiempo mensual del precio de la electricidad en Colombia. Lecturas de Economía, (76), 259-291. http://aprendeenlinea.udea.edu.co/revistas/index. $\mathrm{php} /$ lecturasdeeconomia/article/view/12817

Cavaliere, G. \& Georgiev, I. (2006). A Note on Unit Root Testing in the Presence of Level Shifts. Statistica, 66(1). https://rivista-statistica.unibo.it/article/ viewFile/443/435

Congreso de Colombia. (2001, 27 de sep.). Ley 693 de septiembre 19 de 2001. Por la cual se dictan normas sobre el uso de alcoholes carburantes, se crean estímulos para su producción, comercialización y consumo, y se dictan otras disposiciones. Diario Oficial, (44.564). https://doi.org/10.1098/rstb.2000.0775

Consejo Nacional de Política Económica y Social -CONPES-. (2009). Documento CONPES 3582, 69. https://www.dnp.gov.co/Portals/0/archivos/documentos/ Subdireccion/Conpes/3582.pdf 
Corredor, A. (2017). Empleo y productividad agropecuaria en Colombia. 151-186. https://doi.org/10.18356/784024d8-es

DANE. (2016). Encuesta de empleo directo sector palmero. https://www.dane. gov.co/files/investigaciones/boletines/agropecuario/sector-palmero/bol-empleo-sector-palmero-2016.pdf

DANE. (2019). Encuesta mensual manufacturera -EMM-. https:// www.dane.gov.co/index.php/estadisticas-por-tema/industria/ encuesta-mensual-manufacturera-emm

Delgado, J. E., Salgado, J. J. \& Pérez, R. (2015). Perspectivas de los biocombustibles en Colombia. Revista Ingenierías Universidad de Medellín. https://doi. org/10.22395/rium.v14n27a1

Dickey, D. \& Fuller, W. A. (1979). Distribution of the Estimators for Autoregressive Time Series with a Unit Root. Journal of the American Statistical Association, 74, 427-431. https://doi.org/10.1080/01621459.1979.10482531

DNP. (2008). Lineamientos de política para promover la producción sostenible de biocombustibles en Colombia. Documento Conpes, (3510).

EIA. (2019). EIA Expects Stable U.S. Biofuels Production, Consumption, and Trade through 2020. https://www.eia.gov/todayinenergy/detail.php?id=38532

Elliott G., Rothenberg TJ., Stock JH. (1996). Efficient Tests for an Autoregressive Unit Root. Econometrica. https://doi.org/10.2307/2171846.

FEDEBIOCOMBUSTIBLES. (2019). Federación Nacional de Biocombustibles de Colombia. http://www.fedebiocombustibles.com/nota-web-id-487.htm

FEDESARROLLO. (2012). Evaluación de la política de biocombustibles en Colombia.

García, H. \& Calderón, L. (2012). Evaluación de la política de biocombustibles en Colombia. https://www.repository.fedesarrollo.org.co/handle/11445/338

Lombana, J., Vega, J., Britton, E. \& Herrera, S. (2015). Análisis del sector biodiesel en Colombia y su cadena de suministro. Universidad del Norte. https://dialnet. unirioja.es/servlet/libro?codigo $=579409$ 
Manrique, L. C., Gutiérrez, E. \& González, G. (2008). Computable General Equilibrium (Egc) for the Production of Sugar and Bioethanol in Colombia. Revista de Ingeniería, 28, 108-112. http://www.scielo.org.co/pdf/ring/n28/ n28a15.pdf

Ministerio de Minas y Energía de Colombia. (2009). Resolución 180515 de abril 1 de 2009. Imprenta Nacional.

Ministerio de Minas y Energía. (2019). Reservas de petróleo en Colombia. https:// www.datos.gov.co/Minas-y-Energ-a/Reservas-De-Petroleo/2njd-akei

Neuwahl, F., Löschel, A., Mongelli, I. \& Delgado, L. (2008). Employment Impacts of EU Biofuels Policy: Combining Bottom-up Technology Information and Sectoral Market Simulations in an Input-Output Framework. Ecological Economics, 68(1-2), 447-460. https://doi.org/10.1016/j.ecolecon.2008.04.018

Phillips, P. C. B. \& Perron, P. (1988). Testing for a Unit Root in Time Series Regression. Biometrika, 75(2), 335-346. https://doi.org/10.1093/biomet/75.2.335

Sánchez, M. (2015). Evolución de los biocombustibles en Colombia y su incidencia sobre el precio de los alimentos. Universidad Nacional de Colombia. http:// www.bdigital.unal.edu.co/52262/1/52787408.2016.pdf

Silalertruksa, T., Gheewala, S. H., Hünecke, K. \& Fritsche, U. R. (2012). Biofuels and Employment Effects: Implications for Socio-economic Development in Thailand. Biomass and Bioenergy, 46, 409-418. https://oi.org/10.1016/j. biombioe.2012.07.019

UPME. (2019). Simec Sistema de Información Minero-Energético Colombiano. http://www.simec.gov.co/

Valencia, M. J. \& Cardona, C. A. (2014). The Colombian Biofuel Supply Chains: The Assessment of Current and Promising Scenarios Based on Environmental Goals. Energy Policy. https://doi.org/10.1016/j.enpol.2013.12.021 